\title{
Impact of antenna correlation on the performance of partial relay selection
}

\author{
Nuwan S Ferdinand, Upul Jayasinghe, Nandana Rajatheva* and Matti Latva-aho
}

\begin{abstract}
Antenna correlation is generally viewed as an obstacle to realize the desired performance of a wireless system. In this article, we investigate the performance of partial relay selection in the presence of antenna correlation. We consider both channel state information (csi)-assisted and fixed gain amplify-and-forward (AF) relay schemes. The source and the destination are equipped with multiple antennas communicating via the best first hop signal-to-noise ratio (SNR) relay. We derived the closed form expression for outage probability, average symbol error rate (SER) for both schemes. Further, an exact expression is derived for the ergodic capacity in the csi-assisted relay case and an approximated expression is considered for the fixed gain case. Moreover, we provide simple asymptotic results and show that the diversity order of the system remains unchanged with the effect of antenna correlation for both types of relay schemes.
\end{abstract}

\section{Introduction}

Two-hop amplify-and-forward (AF) relay networks have been investigated extensively in recent research [1-4]. The system with a source and a destination both equipped with multiple antennas communicating via a single antenna relay has received significant interest in most of the previous literature [5-11]. Different transmission and receive techniques were used and use of maximal ratio transmission (MRT) and maximal ratio combining (MRC) were among the most significant ones [5-9]. The analyzes in these cases were carried out with different fading channel environments for performance evaluation.

Antenna correlation is generally considered as a detrimental effect which degrades the system performance. To investigate this loss, several authors have studied the effect of antenna correlation in AF relay schemes. Authors in [7] have analyzed the channel state information (csi)assisted AF relay network under antenna correlation with distinct eigenvalue distribution of correlation matrices and the fixed gain scheme has been considered in [12]. Then the general case of arbitrary distributed correlation matrix structures has been investigated by the authors in [9]. However, these evaluations are limited to the single source, relay and destination scenario.

\footnotetext{
*Correspondence: rrajathe@ee.oulu.fi
}

Department of Communications Engineering, University of Oulu, Oulu, Finland
It has been proven that the use of multiple relays with different selection methods can enhance the diversity and the performance [13-23]. There are several ways of selecting a relay for transmission. One method is referred to as the opportunistic relay selection $[13,14]$ in which the relay with maximum instantaneous end-to-end signal-to-noise ratio (SNR) is considered. Synchronization is very important in this case. Another is the partial relay selection method, which can be carried out in two ways; by selecting either the first-hop relay $[15,19,21]$ or the second hop relay $[13,17,19]$ with the maximum instantaneous SNR. All these studies have been concentrated on the independent and identically distributed fading environments with some considering the effect of feedback delay.

\section{Contribution}

Although authors in the previous literature have studied the AF relay network under the effect of antenna correlation, all these works have been limited to single relay network. Hence, it motivated us to investigate the performance of partial relay selection with the effect of antenna correlation. We consider two types of AF relay schemes; csi-assisted and fixed gain relay. The exact closed form expressions for outage probability and average symbol error rate (SER) are derived for both schemes and an exact ergodic capacity expression is derived for the csi-assisted case and an approximation is found for the other case. Further, we study the system in high SNR and derive simple

\section{Springer}

(c) 2012 Ferdinand et al. licensee Springer. This is an Open Access article distributed under the terms of the Creative Commons Attribution License (http://creativecommons.org/licenses/by/2.0), which permits unrestricted use, distribution, and reproduction in any medium, provided the original work is properly cited. 
asymptotic expressions for outage probability and average SER for both cases. Our asymptotic analysis provide the depth in the system performance and it shows the variation of diversity gain. Finally, we give Monte Carlo simulations to verify our results.

\section{System model}

Consider an AF relay network where a source (S) communicates with a destination (D) via the best relay (R). Both $S$ and $\mathrm{D}$ are equipped with $n_{s}$ and $n_{d}$ antennas, respectively, and relays are equipped with a single antenna. Direct path between source to destination is assumed to be unavailable due to heavy shadowing. The csi is assumed to be available at $\mathrm{S}$. When the csi is available at the transmitter, the optimal transmission scheme is maximal ratio transmission (MRT) [24], hence, S uses MRT as the transmission scheme and destination uses MRC. We consider a system where all the relays are homogeneously located having the same average SNR and we further assume that $S-R_{i} \forall i$ channels are independent of each other. Source uses the csi to find the maximum SNR relay from $L$ number of relays in the first hop as,

$$
\left\|\mathbf{h}_{\mathrm{sm}}\right\|_{F}=\max _{1<i<L}\left\|\mathbf{h}_{\mathrm{si}}\right\|_{F}
$$

where $\|\cdot\|_{F}$ denotes the Frobenius norm and $\mathbf{h}_{\mathrm{si}}$ is the $n_{S} \times 1$ channel vector between $\mathrm{S}-\mathrm{R}$ and the elements of $\mathbf{h}_{\text {si }}$ are modeled as mutually correlated Ralyeigh fading entries. Let $n_{s} \times n_{s}$ correlation matrix at source be $\Phi_{s}$, then $\Phi_{s}=E\left[\mathbf{h}_{\mathrm{si}} \mathbf{h}_{\mathrm{si}}^{\dagger}\right]$, where $E[\cdot]$ and $(\cdot)^{\dagger}$ denote the expectation operator and the Hermitian transpose, respectively. The communication happens in two time slots as presented in numerous literature. During the first time slot, $\mathrm{S}$ transmits the signal $x$ to the selected relay $\mathrm{R}_{\mathrm{m}}$ and the received signal at $R_{m}$ is given as,

$$
y_{r}=\sqrt{P_{s}} \mathbf{h}_{\mathrm{sm}} \mathbf{w}_{s} x+v_{m}
$$

where $P_{s}$ is the transmitted power and $\mathbf{h}_{\mathrm{sm}}$ is given as in (1) and $\mathbf{w}_{s}$ is the MRT weight vector which is defined as $\mathbf{w}_{s}=\mathbf{h}_{\mathrm{sm}}^{\dagger} /\left\|\mathbf{h}_{\mathrm{sm}}\right\|_{F}$. Additive white Gaussian noise (AWGN) component with $V_{m}$ variance at $\mathrm{R}_{\mathrm{m}}$ is denoted as $v_{m}$. Then $R_{m}$ multiplies the received signal by gain $G$ and transmits to the $\mathrm{D}$ and the received signal at $\mathrm{D}$ is given as,

$$
\mathbf{y}_{d}=\mathbf{h}_{\mathrm{md}} G \sqrt{P_{r}}\left(\sqrt{P_{s}} \mathbf{h}_{\mathrm{sm}} \mathbf{w}_{s} x+v_{m}\right)+\mathbf{v}_{d}
$$

where $G$ is defined differently for the two relay schemes and is given in the next section. $P_{r}$ is the transmitted power at $R_{m}$ and $1 \times n_{d}$ channel vector between $R_{m}-D$ is $\mathbf{h}_{\text {md }}$ and its elements are mutually correlated such that the correlation matrix at $\mathrm{D}$ is $\Phi_{d}=E\left[\mathbf{h}_{\mathrm{md}} \mathbf{h}_{\mathrm{md}}^{\dagger}\right] . \mathbf{v}_{d}$ is noise vector at D and it elements are AWGN with $V_{d}$ variance. Now D performs MRC to obtain the signal as,

$$
y_{d}=\mathbf{w}_{d} \mathbf{h}_{\mathrm{md}} G \sqrt{P_{r}}\left(\sqrt{P_{s}} \mathbf{h}_{\mathrm{sm}} \mathbf{w}_{s} x+v_{m}\right)+\mathbf{w}_{d} \mathbf{v}_{d}
$$

where $\mathbf{w}_{d}=\mathbf{h}_{\mathrm{md}}^{\dagger} /\left\|\mathbf{h}_{\mathrm{md}}\right\|_{F}$ is the MRC weight vector. Now after some mathematical manipulations, we obtain the end-to-end SNR as,

$$
\gamma_{e}=\frac{\frac{P_{s}}{V_{m}}\left\|\mathbf{h}_{\mathrm{sm}}\right\|_{F}^{2} \frac{P_{r}}{V_{d}}\left\|\mathbf{h}_{\mathrm{md}}\right\|_{F}^{2}}{\frac{P_{r}}{V_{d}}\left\|\mathbf{h}_{\mathrm{md}}\right\|_{F}+\frac{1}{G^{2} V_{m}}}
$$

Notation: Let $\rho_{1}=\frac{P_{s}}{V_{m}}$ and $\rho_{2}=\frac{P_{r}}{V_{d}}$ and define $\gamma_{1}=\left\|\mathbf{h}_{\text {sm }}\right\|_{F}^{2} \rho_{1}$ and $\gamma_{2}=\left\|\mathbf{h}_{\text {md }}\right\|_{F}^{2} \rho_{2}$. Let the distinct eigenvalues of the correlation matrix at source $\Phi_{s}$ be $\phi_{1}, \phi_{2}, \ldots, \phi_{n_{s}}$ and those of the correlation matrix at the destination $\Phi_{d}$ be $\sigma_{1}, \sigma_{2}, \ldots, \sigma_{n_{d}}$.

\section{Statistics of SNRs}

We can derive the probability density function (pdf) of $\gamma_{2}$ as $[25,26]$,

$$
p_{\gamma_{2}}(z)=\sum_{u=1}^{n_{d}} \frac{\sigma_{u}^{n_{d}-2}}{\prod_{k=1, k \neq u}^{n_{d}}\left(\sigma_{u}-\sigma_{k}\right)} \frac{\exp \left(\frac{-z}{\rho_{2} \sigma_{u}}\right)}{\rho_{2}}
$$

and the cumulative density function (cdf) of $\gamma_{2}$ can be derived using $F_{\gamma_{2}}(\Lambda)=\int_{0}^{\Lambda} p_{\gamma_{2}}(z) d z$ with the help of ([27], Equation 2.321.2) as,

$$
F_{\gamma_{2}}(z)=1-\sum_{u=1}^{n_{d}} \frac{\sigma_{u}^{n_{d}-1}}{\prod_{k=1, k \neq u}^{n_{d}}\left(\sigma_{u}-\sigma_{k}\right)} \exp \left(\frac{-z}{\rho_{2} \sigma_{u}}\right)
$$

Now, we derive the cdf of $\gamma_{1}$ as,

$$
\begin{aligned}
F_{\gamma_{1}}(z)= & 1-\sum_{w_{o}=1}^{L}(-1)^{w_{o}+1}\left(\begin{array}{c}
L \\
w_{o}
\end{array}\right) \sum_{w_{1}=0}^{w_{o}} \sum_{w_{2}=0}^{w_{1}} \ldots \sum_{w_{n_{s}-1}=0}^{w_{n_{s}-2}} \prod_{i=1, w_{n_{s}}=0}^{n_{s}} \\
& \times\left[\frac{\phi_{i}^{n_{s}-1}}{\prod_{k=1, k \neq i}^{n_{s}}\left(\phi_{i}-\phi_{k}\right)}\right]^{w_{i-1}-w_{i}}\left(\begin{array}{c}
w_{i-1} \\
w_{i}
\end{array}\right) \\
& \times \exp \left(-\sum_{i=1, w_{n_{s}=0}}^{n_{s}} \frac{\left(w_{i-1}-w_{i}\right) z}{\rho_{1} \phi_{i}}\right)
\end{aligned}
$$

Proof. See Appendix 1.

\section{Channel state information assisted relay}

Here the relay uses the csi to amplify the received signal. It has been proven that the csi-assisted relay outperforms the fixed gain relay in general. However, it has a higher complexity in implementation when compared to the fixed gain one. In this section, we derive exact expressions 
for outage probability, average SER and ergodic capacity. First, we select the gain $G$ for csi-assisted relay as,

$$
G=\sqrt{\frac{1}{P_{s}\left\|\mathbf{h}_{\mathrm{sm}}\right\|_{F}^{2}+V_{m}}}
$$

Then the end-to-end SNR given in (5) can be rewritten as,

$$
\gamma_{e}=\frac{\gamma_{1} \gamma_{2}}{\gamma_{1}+\gamma_{2}+c} \approx \frac{\gamma_{1} \gamma_{2}}{\gamma_{1}+\gamma_{2}}
$$

where $c=1$ for exact end-to-end SNR. The approximation holds for the medium to high SNR and it provides a tight upper bound, we use the exact SNR to derive the outage probability and ergodic capacity, and use the approximation for average SER.

\section{Outage probability: csi-assisted relay}

The outage probability is the probability that $\gamma_{e}$ drops below a predefined threshold $\Lambda$ and it is mathematically represented as,

$$
P_{\text {out }}=\operatorname{Pr}\left(\gamma_{e}<\Lambda\right)=\operatorname{Pr}\left(\frac{\gamma_{1} \gamma_{2}}{\gamma_{1}+\gamma_{2}+c}<\Lambda\right)
$$

We can derive the exact closed form expression for outage probability as,

$$
\begin{aligned}
F_{\gamma_{e}}(\Lambda)= & 1-2 \sum_{w_{0}=1}^{L}(-1)^{w_{0}+1}\left(\begin{array}{c}
L \\
w_{0}
\end{array}\right) \sum_{w_{1}=0}^{w_{0}} \sum_{w_{2}=0}^{w_{1}} \ldots \sum_{w_{n_{s}-1}=0}^{w_{n_{s}-2}} \\
& \times\left[\prod_{i=1, w_{n_{s}}=0}^{n_{s}}\left[\frac{\phi_{i}^{n_{s}-1}}{\prod_{k=1, k \neq i}^{n_{s}}\left(\phi_{i}-\phi_{k}\right)}\right]^{w_{i-1}-w_{i}}\right. \\
& \left.\times\left(\begin{array}{c}
w_{i-1} \\
w_{i}
\end{array}\right)\right] \sum_{u=1}^{n_{d}} \frac{\sigma_{u}^{n_{d}-1}}{\prod_{k=1, k \neq u}^{n_{d}}\left(\sigma_{u}-\sigma_{k}\right)} \\
& \times \sqrt{\sum_{i=1, w_{n_{s}}=0}^{n_{s}} \frac{\left(w_{i-1}-w_{i}\right)}{\rho_{1} \phi_{i} \rho_{2} \sigma_{u}} \exp \left(-\frac{\Lambda}{\rho_{2} \sigma_{u}}\right.} \\
& \left.-\sum_{i=1, w_{n_{s}}=0}^{n_{s}} \frac{\left(w_{i-1}-w_{i}\right) \Lambda}{\rho_{1} \phi_{i}}\right) \sqrt{\Lambda(\Lambda+c)} \\
& \times \mathrm{K}_{1}\left(2 \sqrt{\sum_{i=1, w_{n_{s}}=0}^{n_{s}} \frac{\left(w_{i-1}-w_{i}\right) \Lambda(\Lambda+c)}{\rho_{1} \rho_{2} \phi_{i} \sigma_{u}}}\right)
\end{aligned}
$$

where $K_{1}(\cdot)$ is the first order modified Bessel function of first kind.
Proof. Appendix 1.

\section{Outage probability: approximation}

We simplify (12) by substituting $c=0$ to obtain the tight upper bound as given in (11) as,

$$
\begin{aligned}
F_{\gamma_{e}}(\Lambda)= & 1-2 \sum_{w_{0}=1}^{L}(-1)^{w_{0}+1}\left(\begin{array}{c}
L \\
w_{0}
\end{array}\right) \sum_{w_{1}=0}^{w_{0}} \sum_{w_{2}=0}^{w_{1}} \ldots \sum_{w_{n_{s}-1}=0}^{w_{n_{s}-2}} \\
& \times\left[\prod_{i=1, w_{n_{s}}=0}^{n_{s}}\left[\frac{\phi_{i}^{n_{s}-1}}{\prod_{k=1, k \neq i}^{n_{s}}\left(\phi_{i}-\phi_{k}\right)}\right]^{w_{i-1}-w_{i}}\right. \\
& \left.\times\left(\begin{array}{c}
w_{i-1} \\
w_{i}
\end{array}\right)\right] \sum_{u=1}^{n_{d}} \frac{\sigma_{u}^{n_{d}-1}}{\prod_{k=1, k \neq u}^{n_{d}}\left(\sigma_{u}-\sigma_{k}\right)} \\
& \times \sqrt{\sum_{i=1, w_{n_{s}}=0}^{n_{s}} \frac{\left(w_{i-1}-w_{i}\right)}{\rho_{1} \phi_{i} \rho_{2} \sigma_{u}} \Lambda \exp \left(-\frac{\Lambda}{\rho_{2} \sigma_{u}}\right.} \\
& \left.-\sum_{i=1, w_{n_{s}}=0}^{n_{s}} \frac{\left(w_{i-1}-w_{i}\right) \Lambda}{\rho_{1} \phi_{i}}\right) \\
& \times \mathrm{K}_{1}\left(2 \Lambda \sqrt{\sum_{i=1, w_{n_{s}}=0}^{n_{s}} \frac{\left(w_{i-1}-w_{i}\right)}{\rho_{1} \rho_{2} \phi_{i} \sigma_{u}}}\right)
\end{aligned}
$$

\section{Average SER}

In this section, we derive the closed form expressions for average SER $\left(P_{\text {ser }}\right)$ which is valid for several modulation schemes. According to technical literature $P_{\text {ser }}$ is defined as,

$$
P_{\text {ser }}=E\left[a Q\left(\sqrt{2 b \gamma_{e}}\right)\right]
$$

where $E[\cdot]$ is the expectation operator and $Q($.$) is$ the Gaussian $\mathrm{Q}$ function. The modulation schemes is defined by $a, b$, mainly BPSK $(a=1, b=1)$, M-ary PAM $\left(a=\frac{2(M-1)}{M}, b=\frac{3}{M^{2}-1}\right)$ and M-PSK $\left(a=2, b=\sin ^{2} \frac{\pi}{M}\right)$ $[28,29]$. Carrying out integration by parts of (14), we obtain,

$$
P_{\text {ser }}=\frac{a \sqrt{b}}{2 \sqrt{\pi}} \int_{0}^{\infty} \frac{F_{\gamma_{e}}(z)}{\sqrt{z}} \exp (-b z) d z
$$

Now, we substitute $F_{\gamma_{e}}(\Lambda)$ in (13) to (15) and perform the integration with the help of ([27], Equation 6.621.3), to obtain the closed form expression for the average SER of $\gamma_{e}$ as, 


$$
P_{\text {ser }}=\frac{a}{2}-4 a \sqrt{b} \sum_{w_{0}=1}^{L}(-1)^{w_{0}+1}\left(\begin{array}{c}
L \\
w_{0}
\end{array}\right) \sum_{w_{1}=0}^{w_{0}} \sum_{w_{2}=0}^{w_{1}} \ldots \sum_{w_{n_{s}-1}=0}^{w_{n_{s}-2}}
$$

$$
\begin{aligned}
& {\left[\prod_{i=1, w_{n_{s}}=0}^{n_{s}}\left[\frac{\phi_{i}^{n_{s}-1}}{\prod_{k=1, k \neq i}^{n_{s}}\left(\phi_{i}-\phi_{k}\right)}\right]^{w_{i-1}-w_{i}}\left(\begin{array}{c}
w_{i-1} \\
w_{i}
\end{array}\right)\right]} \\
& \quad \times \sum_{u=1}^{n_{d}} \frac{\frac{\sigma_{u}^{n_{d}-1}}{\prod_{k=1, k \neq u}^{n_{d}}\left(\sigma_{u}-\sigma_{k}\right)} \sum_{i=1, w_{n_{s}}=0}^{n_{s}} \frac{\left(w_{i-1}-w_{i}\right)}{\rho_{1} \phi_{i} \rho_{2} \sigma_{u}} \Gamma\left(\frac{5}{2}\right) \Gamma\left(\frac{1}{2}\right)}{\left(\frac{1}{\rho_{2} \sigma_{u}}+\sum_{i=1, w_{n_{s}}=0}^{n_{s}} \frac{\left(w_{i-1}-w_{i}\right)}{\rho_{1} \phi_{i}}+b+\beta_{1}\right)^{\frac{5}{2}}} \\
& \quad \times F\left(\frac{5}{2}, \frac{3}{2} ; 2 ; \frac{\frac{1}{\rho_{2} \sigma_{u}}+\sum_{i=1, w_{n_{s}}=0}^{n_{s}} \frac{\left(w_{i-1}-w_{i}\right)}{\rho_{1} \phi_{i}}+b-\beta_{1}}{\frac{1}{\rho_{2} \sigma_{u}}+\sum_{i=1, w_{n_{s}}=0}^{n_{s}} \frac{\left(w_{i-1}-w_{i}\right)}{\rho_{1} \phi_{1}}+b+\beta_{1}}\right)
\end{aligned}
$$

where

$$
\beta_{1}=2 \sqrt{\sum_{i=1, w_{n_{s}}=0}^{n_{s}} \frac{\left(w_{i-1}-w_{i}\right)}{\rho_{1} \phi_{i} \rho_{2} \sigma_{u}}}
$$

where $F(\mu, v ; a ; b)$ is the Gauss hypergeometric function defined in ([27], Equation 9.10-9.13) and $\Gamma(\cdot)$ represents the Gamma function.

\section{Ergodic capacity}

The exact closed form expression for ergodic capacity has a significant importance since it has not been derived even for a single user relay network with antenna correlation. The ergodic capacity $\left(C_{\text {erg }}\right)$ can be mathematically expressed as,

$$
C_{\text {erg }}=\mathrm{E}\left[R \log _{2}\left(1+\gamma_{e}\right)\right]
$$

Closed form expression for $C_{\text {erg }}$ can be derived as,

$$
C_{\text {erg }}=\frac{R}{\ln 2}\left[\chi_{1}+\chi_{2}-\chi_{3}\right]
$$

where

$$
\begin{aligned}
\chi_{1}= & \sum_{w_{0}=1}^{L}(-1)^{w_{0}+1}\left(\begin{array}{c}
L \\
w_{0}
\end{array}\right) \sum_{w_{1}=0}^{w_{0}} \sum_{w_{2}=0}^{w_{1}} \ldots \sum_{w_{n_{s}-1}=0}^{w_{n_{s}-2}}\left[\prod_{i=1, w_{n_{s}}=0}^{n_{s}}\right. \\
& \left.\times\left[\frac{\phi_{i}^{n_{s}-1}}{\prod_{k=1, k \neq i}^{n_{s}}\left(\phi_{i}-\phi_{k}\right)}\right]^{w_{i-1}-w_{i}}\left(\begin{array}{c}
w_{i-1} \\
w_{i}
\end{array}\right)\right] e^{\frac{\beta_{2}}{\rho_{1}}} \mathrm{E}_{1}\left(\frac{\beta_{2}}{\rho_{1}}\right)
\end{aligned}
$$

where $\mathrm{E}_{1}(z)=\int_{z}^{\infty} \frac{e^{-x}}{x} d x$ is the exponential integral. $\chi_{2}$ is given as,

$$
\chi_{2}=\sum_{u=1}^{n_{d}} \frac{\sigma_{u}^{n_{d}-1}}{\prod_{k=1, k \neq u}^{n_{d}}\left(\sigma_{u}-\sigma_{k}\right)} e^{\frac{1}{\rho_{2} \sigma_{u}}} \mathrm{E}_{1}\left(\frac{1}{\rho_{2} \sigma_{u}}\right)
$$

and

$$
\begin{aligned}
& \chi_{3}=\left\{\begin{array}{l}
\xi_{1}, \frac{1}{\rho_{2} \sigma_{u}} \neq \frac{\beta_{2}}{\rho_{1}} \\
\xi_{2}, \frac{1}{\rho_{2} \sigma_{u}}=\frac{\beta_{2}}{\rho_{1}}
\end{array}\right. \\
& \xi_{1}=\sum_{u=1}^{n_{d}} \frac{\sigma_{u}^{n_{d}-1}}{\prod_{k=1, k \neq u}^{n_{d}}\left(\sigma_{u}-\sigma_{k}\right)} \sum_{w_{0}=1}^{L}(-1)^{w_{0}+1}\left(\begin{array}{c}
L \\
w_{0}
\end{array}\right) \sum_{w_{1}=0}^{w_{0}} \\
& \times \sum_{w_{2}=0}^{w_{1}} \ldots \sum_{w_{n_{s}-1}=0}^{w_{n_{s}-2}}\left(\frac{1}{\rho_{2} \sigma_{u}}-\frac{\beta_{2}}{\rho_{1}}\right)^{-1} \\
& \times\left[\prod_{i=1, w_{n_{s}}=0}^{n_{s}}\left[\frac{\phi_{i}^{n_{s}-1}}{\prod_{k=1, k \neq i}^{n_{s}}\left(\phi_{i}-\phi_{k}\right)}\right]^{w_{i-1}-w_{i}}\left(\begin{array}{c}
w_{i-1} \\
w_{i}
\end{array}\right)\right] \\
& \times\left[\frac{\mathrm{E}_{1}\left(\frac{\beta_{2}}{\rho_{1}}\right)}{\rho_{2} \sigma_{u} e^{-\frac{\beta_{2}}{\rho_{1}}}}-\frac{\beta_{2} \mathrm{E}_{1}\left(\frac{1}{\rho_{2} \sigma_{2}}\right)}{\rho_{1} e^{-\frac{1}{\rho_{2} \sigma_{u}}}}\right] \\
& \xi_{2}=\sum_{u=1}^{n_{d}} \frac{\sigma_{u}^{n_{d}-1}}{\prod_{k=1, k \neq u}^{n_{d}}\left(\sigma_{u}-\sigma_{k}\right)} \sum_{w_{0}=1}^{L}(-1)^{w_{0}+1}\left(\begin{array}{c}
L \\
w_{0}
\end{array}\right) \sum_{w_{1}=0}^{w_{0}} \\
& \times \sum_{w_{2}=0}^{w_{1}} \ldots \sum_{w_{n_{s}-1}=0}^{w_{n_{s}-2}} \\
& \times\left[\prod_{i=1, w_{n_{s}}=0}^{n_{s}}\left[\frac{\phi_{i}^{n_{s}-1}}{\prod_{k=1, k \neq i}^{n_{s}}\left(\phi_{i}-\phi_{k}\right)}\right]^{w_{i-1}-w_{i}}\left(\begin{array}{c}
w_{i-1} \\
w_{i}
\end{array}\right)\right] \\
& \times\left[1+\left(1-\frac{1}{\rho_{2} \sigma_{u}}\right) e^{\frac{1}{\rho_{2} \sigma_{u}}} \mathrm{E}_{1}\left(\frac{1}{\rho_{2} \sigma_{u}}\right)\right]
\end{aligned}
$$

where

$$
\beta_{2}=\sum_{i=1, w_{n_{s}}=0}^{n_{s}} \frac{w_{i-1}-w_{i}}{\phi_{i}}
$$

Proof. Appendix 2

\section{High SNR analysis}

Here we derive the high SNR expressions for the outage probability and the average SER. Let $z=\frac{\Lambda}{\rho_{1}}$ and $\rho_{2}=\mu \rho_{1}$. 


\section{High SNR outage probability}

We rewrite the (7) by expanding the $e^{\frac{-z}{\sigma_{u}}}$ using Maclaurin series as follows,

$F_{\gamma_{2}}(z)=1-\sum_{u=1}^{n_{d}} \frac{\sigma_{u}^{n_{d}-1}}{\prod_{k=1, k \neq u}^{n_{d}}\left(\sigma_{u}-\sigma_{k}\right)} \sum_{s=0}^{\infty}\left(-\frac{z}{\mu \sigma_{u}}\right)^{s}$

It is observed at high SNR the lower order terms $\left(z^{n} ; n<\right.$ $n_{s}$ ) sum to zero, hence, by collecting the higher order terms, we can express (28) in high SNR as,

$$
F_{\gamma_{2}}(z)=\sum_{u=1}^{n_{d}} \frac{\sigma_{u}^{n_{d}-1}}{\prod_{k=1, k \neq u}^{n_{d}}\left(\sigma_{u}-\sigma_{k}\right)}\left(-\frac{z}{\mu \sigma_{u}}\right)^{n_{d}}+o\left(z^{n_{d}+1}\right)
$$

We can further simplify (29) as,

$$
F_{\gamma_{2}}(z)=\frac{z^{n_{d}}}{\mu^{n_{d}} n_{d} ! \prod_{u=1}^{n_{d}} \sigma_{u}}+o\left(z^{n_{d}+1}\right)
$$

Similarly, we can derive the $F_{\gamma_{1}}(z)$ in high SNR as

$$
F_{\gamma_{1}}(z)=\left[\frac{z^{n_{s}}}{n_{s} ! \prod_{i=1}^{n_{s}} \phi_{i}}+o\left(z^{n_{s}+1}\right)\right]^{L}
$$

Simplification yields,

$$
F_{\gamma_{1}}(z)=\frac{z^{n_{s} L}}{\left(n_{s} ! \prod_{i=1}^{n_{s}} \phi_{i}\right)^{L}}+o\left(z^{n_{s} L+1}\right)
$$

Following the same procedure as in ([30], Equations (A.09) and (A.10)), we can obtain the high SNR expression for $F_{\gamma_{\text {end }}}(z)$ as,

$$
F_{\gamma_{e}}(z)= \begin{cases}\psi_{1} z^{L n_{s}}+o\left(z^{L n_{s}+1}\right), & L n_{s}<n_{d} \\ \psi_{2} z^{n_{d}}+o\left(z^{n_{d}+1}\right), & L n_{s}>n_{d} \\ \psi_{3} z^{n}+o\left(z^{n+1}\right), & n_{s}=L n_{d}=n\end{cases}
$$

where,

$$
\begin{aligned}
& \psi_{1}=\left[\frac{1}{n_{s} ! \prod_{i=1}^{n_{s}} \phi_{i}}\right]^{L} \\
& \psi_{2}=\frac{1}{\mu^{n_{d}} n_{d} ! \prod_{u=1}^{n_{d}} \sigma_{u}} \\
& \psi_{3}=\psi_{1}+\psi_{2}
\end{aligned}
$$

It is observed from (33) that the diversity gain $G_{d}=$ $\min \left[L n_{s}, n_{d}\right]$. This shows that the performance of the system is dominated by one of the single links unless $L n_{s}=$ $n_{d}$, hence, in order to fully utilize the resources, we like to keep $L n_{s}=n_{d}$. It is observed here that the diversity gain of the system is not affected by the antenna correlation. However, we have to note that this condition is only true for the case where the correlation matrices have full rank.

\section{High SNR average SER}

Average SER in high SNR can be obtained using [31]

$P_{s e r}^{\infty}=\frac{2^{t} a \psi \Gamma\left(t+\frac{3}{2}\right)\left(2 b \rho_{1}\right)^{-(t+1)}}{\sqrt{\pi}}+o\left(\rho_{1}^{-(t+1)}\right)$

where $a$ and $b$ define the modulation scheme and $\psi$ is given as in (34)-(36). $t=\min \left[L n_{s}, n_{d}\right]-1$ and diversity gain $G_{d}=t+1$.

\section{Fixed gain relay}

In this section, we investigate the end to end performance of a dual-hop fixed gain network with multiple relays in the presence of antenna correlation at both ends. We derive closed-form expressions for outage probability, average SER, generalized moments of the end-to-end SNR and ergodic capacity. The asymptotic behavior of the system under high SNR is also considered. After performing some algebraic manipulations of (5), the end-to-end SNR for fixed gain can be expressed as,

$$
\gamma_{e}=\frac{\gamma_{1} \gamma_{2}}{\gamma_{2}+C}
$$

According to literature, there are two common techniques for selecting a fixed gain $G$ in (38). If the gain is selected as,

$$
G=\sqrt{E_{|| \mathbf{h}_{\mathrm{sm}} \|_{F}^{2}}\left[\frac{1}{P_{s} \mid \mathbf{h}_{\mathrm{sm}} \|_{F}^{2}+V_{m}}\right]}
$$

then the constant $C$ given by

$$
C=\left(E_{\gamma_{1}}\left[\frac{1}{\left(\gamma_{1}+1\right)}\right]\right)^{-1}
$$

If the gain is selected as,

$$
G=\sqrt{\frac{1}{P_{s} E_{|| \mathbf{h}_{\mathrm{sm}} \|_{F}^{2}[}\left[\mid \mathbf{h}_{\mathrm{sm}} \|_{F}^{2}\right]+V_{m}}}
$$

the constant $C$ given by

$$
C=E_{\gamma_{1}}\left(\gamma_{1}\right)+1
$$

\section{Outage probability}

If outage probability is denoted by $P_{\text {out }}$, then

$$
P_{\text {out }}=P\left(\gamma_{e}<\Lambda\right)=P\left(\frac{\gamma_{1} \gamma_{2}}{\gamma_{2}+C}<\Lambda\right)
$$


The closed form expression for outage probability can be derived as,

$$
\begin{aligned}
F_{\gamma_{e}}(\Lambda)= & 1-2 \sum_{w_{o}=1}^{L}(-1)^{w_{o}+1}\left(\begin{array}{c}
L \\
w_{o}
\end{array}\right) \sum_{w_{1}=0}^{w_{o}} \sum_{w_{2}=0}^{w_{1}} \ldots \sum_{w_{n_{s}-1}=0}^{w_{n_{s}-2}} \\
& \times \prod_{v=1, w_{n_{s}}=0}^{n_{s}}\left[\frac{\phi_{v}^{n_{s}-1}}{\prod_{k=1, k \neq v}^{n_{s}}\left(\phi_{v}-\phi_{k}\right)}\right]^{w_{v-1}-w_{v}} \\
& \times\left(\begin{array}{c}
w_{v-1} \\
w_{v}
\end{array}\right) \sum_{u=1}^{n_{d}} \frac{\sigma_{u}^{n_{d}-1}}{\prod_{k=1, k \neq i}^{n_{d}}\left(\sigma_{u}-\sigma_{k}\right)} \\
& \times \sqrt{\sum_{i=1, w_{n_{s}=0}^{n_{s}}} \frac{\left(w_{i-1}-w_{i}\right)}{\rho_{1} \phi_{i} \rho_{2} \sigma_{u}} \sqrt{\Lambda C}} \\
& \times \exp \left(-\sum_{i=1, w_{n_{s}=0}}^{n_{s}} \frac{\left(w_{i-1}-w_{i}\right) \Lambda}{\rho_{1} \phi_{i}}\right) \\
& \times K_{1}\left(2 \sqrt[\sum_{i=1, w_{n_{s}=0}}^{n_{S}} \frac{\left(w_{i-1}-w_{i}\right) \Lambda C}{\rho_{1} \rho_{2} \phi_{i} \sigma_{u}}]{ }\right)
\end{aligned}
$$

where $K_{1}($.$) is 1$ st order modified Bessel function of second type and $C$ is the fixed gain.

\section{Proof. Appendix 3}

From Equation (8), cdf of $\gamma_{1}$ can be written as,

$$
F_{\gamma_{1}}(z)=1-\alpha_{4} \exp \left(-z \beta_{4}\right)
$$

Where,

$$
\begin{aligned}
\alpha_{4}= & \sum_{w_{o}=1}^{L}(-1)^{w_{o}+1}\left(\begin{array}{c}
L \\
w_{o}
\end{array}\right) \sum_{w_{1}=0}^{w_{o}} \sum_{w_{2}=0}^{w_{1}} \ldots \sum_{w_{n_{s}-1}=0}^{w_{n_{s}-2}} \prod_{v=1, w_{n_{s}}=0}^{n_{s}} \\
& \times\left[\frac{\phi_{v}^{n_{s}-1}}{\prod_{k=1, k \neq v}^{n_{s}}\left(\phi_{v}-\phi_{k}\right)}\right]^{w_{v-1}-w_{v}}\left(\begin{array}{c}
w_{v-1} \\
w_{v}
\end{array}\right)
\end{aligned}
$$

and

$$
\beta_{4}=\exp \left(-\sum_{i=1, w_{n_{s}=0}}^{n_{s}} \frac{\left(w_{i-1}-w_{i}\right) z}{\rho_{1} \phi_{i}}\right)
$$

Case 1. Using ([9], Equation 46) the expected value for random variable $\gamma_{1}$ is calculated as,

$$
E\left[\gamma_{1}\right]=\frac{\alpha_{4}}{\beta_{4}}
$$

Substituting to (42), the closed form expression for fixed gain can be derived as,

$$
C=\frac{\alpha_{4}}{\beta_{4}}+1
$$

where $\alpha_{4}$ and $\beta_{4}$ are as mentioned in (46) and (47), respectively.

Case 2.

$$
\begin{aligned}
E\left[\frac{1}{\gamma_{1}+1}\right] & =\int_{0}^{\infty} \frac{1}{\gamma_{1}+1} \frac{d F_{\gamma_{1}}}{d z} d \gamma_{1} \\
& =\alpha_{4} \beta_{4}\left[-\exp \left(\beta_{4}\right) \operatorname{Ei}\left(-\beta_{4}\right)\right]
\end{aligned}
$$

Substituting to (40)

$$
C=\left[\alpha_{4} \beta_{4}\left(-\exp \left(\beta_{4}\right) E i\left(-\beta_{4}\right)\right)\right]^{-1}
$$

where $\mathrm{Ei}(\cdot)$ is the exponential integral.

\section{Average SER}

Then substituting $F_{\gamma_{e}}(z)$ from (44) to (15) and through the mathematical simplification with the help of ([27], Equation 6.614.5), the closed form expression for SER is obtained.

$$
\begin{aligned}
& P_{\text {ser }}=\frac{a}{2}-\frac{a \sqrt{b}}{4} \sum_{w_{o}=1}^{L}(-1)^{w_{o}+1}\left(\begin{array}{c}
L \\
w_{o}
\end{array}\right) \sum_{w_{1}=0}^{w_{o}} \sum_{w_{2}=0}^{w_{1}} \ldots \sum_{w_{n_{S}-1}=0}^{w_{n_{S}-2}} \\
& \times \prod_{v=1, w_{n_{s}}=0}^{n_{s}}\left[\frac{\phi_{\nu}^{n_{s}-1}}{\prod_{k=1, k \neq v}^{n_{s}}\left(\phi_{v}-\phi_{k}\right)}\right]^{w_{v-1}-w_{v}}\left(\begin{array}{c}
w_{v-1} \\
w_{v}
\end{array}\right) \\
& \times \sum_{u=1}^{n_{d}} \frac{\frac{\sigma_{u}^{n_{d}-1}}{\prod_{k=1, k \neq i}^{n_{d}}\left(\sigma_{u}-\sigma_{k}\right)} \sum_{i=1, w_{n_{s}=0}}^{n_{s}} \frac{\left(w_{i-1}-w_{i}\right) C}{\rho_{1} \phi_{i} \rho_{2} \sigma_{u}}}{\left(\sum_{i=1, w_{n_{s}=0}=0}^{n_{s}} \frac{\left(w_{i-1}-w_{i}\right)}{\rho_{1} \phi_{i}}+b\right)^{3 / 2}} \\
& \times \exp \left(0.5 \frac{\sum_{i=1, w_{n_{s}=0}}^{n_{s}} \frac{\left(w_{i-1}-w_{i}\right) C}{\rho_{1} \phi_{i} \rho_{2} \sigma_{u}}}{\sum_{i=1, w_{n_{s}=0}}^{n_{s}} \frac{\left(w_{i-1}-w_{i}\right)}{\rho_{1} \phi_{i}}+b}\right) \\
& \times\left[K_{1}\left(0.5 \frac{\sum_{i=1, w_{n_{s}=0}}^{n_{s}} \frac{\left(w_{i-1}-w_{i}\right) C}{\rho_{1} \phi_{i} \rho_{2} \sigma_{u}}}{\sum_{i=1, w_{n_{s}=0}}^{n_{s}} \frac{\left(w_{i-1}-w_{i}\right)}{\rho_{1} \phi_{i}}+b}\right)\right. \\
& \left.-K_{0}\left(0.5 \frac{\sum_{i=1, w_{n_{s}=0}}^{n_{s}} \frac{\left(w_{i-1}-w_{i}\right) C}{\rho_{1} \phi_{i} \rho_{2} \sigma_{u}}}{\sum_{i=1, w_{n_{s}=0}}^{n_{s}} \frac{\left(w_{i-1}-w_{i}\right)}{\rho_{1} \phi_{i}}+b}\right)\right]
\end{aligned}
$$

where $K_{1}($.$) and K_{0}($.$) are the 1$ st and 2 nd order modified Bessel functions of second type, respectively. 


\section{Generalized moments of SNR}

In this section, we derive closed form expression for generalized moments of $\gamma_{e}$ which is essential to the obtain ergodic capacity and the performance evaluation of the system using the average output SNR and the degree of fading. Substituting (44) into ([9], Equation 46) and after some mathematical manipulations with the help of ([27], Equation 6.643.3), we obtain

$$
\begin{aligned}
& E\left(\gamma_{e}^{h}\right)=h \Gamma(h+1) \Gamma(h) \sum_{w_{o}=1}^{L}(-1)^{w_{o}+1}\left(\begin{array}{c}
L \\
w_{o}
\end{array}\right) \sum_{w_{1}=0}^{w_{o}} \sum_{w_{2}=0}^{w_{1}} \ldots \\
& \times \sum_{w_{n_{s}-1}=0}^{w_{n_{s}-2}} \prod_{v=1, w_{n_{s}}=0}^{n_{s}}\left[\frac{\phi_{v}^{n_{s}-1}}{\prod_{k=1, k \neq v}^{n_{s}}\left(\phi_{v}-\phi_{k}\right)}\right]^{w_{v-1}-w_{v}} \\
& \times\left(\begin{array}{c}
w_{v-1} \\
w_{v}
\end{array}\right) \sum_{u=1}^{n_{d}} \frac{\sigma_{u}^{n_{d}-1}}{\prod_{k=1, k \neq i}^{n_{d}}\left(\sigma_{u}-\sigma_{k}\right)} \\
& \times \exp \left(0.5 \frac{\sum_{i=1, w_{n_{s}=0}}^{n_{s}} \frac{\left(w_{i-1}-w_{i}\right) C}{\rho_{1} \phi_{i} \rho_{2} \sigma_{u}}}{\sum_{i=1, w_{n_{s}=0}}^{n_{s}} \frac{\left(w_{i-1}-w_{i}\right)}{\rho_{1} \phi_{i}}+b}\right) \\
& \times\left(\sum_{i=1, w_{n_{s}=0}}^{n_{s}} \frac{\left(w_{i-1}-w_{i}\right)}{\rho_{1} \phi_{i}}\right)^{-h} \\
& \times W_{-h, 0.5}\left(0.5 \frac{\sum_{i=1, w_{n_{s}=0}}^{n_{s}} \frac{\left(w_{i-1}-w_{i}\right) C}{\rho_{1} \phi_{i} \rho_{2} \sigma_{u}}}{\sum_{i=1, x_{n_{s}=0}}^{n_{s}} \frac{\left(w_{i-1}-w_{i}\right)}{\rho_{1} \phi_{i}}}\right)
\end{aligned}
$$

where $W_{k, \mu}($.$) is the whittaker function defined in ([27],$ Equation 9.220.4).

\section{Ergodic capacity}

We derive a closed form expression for the ergodic capacity in multi relay network which is significant in determining the system performance especially in a correlated environment. Based on the literature ergodic capacity can be expressed as follows,

$$
C_{\mathrm{erg}}=\frac{1}{2} E_{\gamma_{e}}\left[\log _{2}\left(1+\gamma_{e}\right)\right]
$$

applying the expectation operator as in (54), an approximated result can be obtained as given below ([18], Equation 6)

$$
C_{\mathrm{erg}}=\frac{1}{2} \log _{2}(e)\left[\ln \left(1+E\left[\gamma_{e}\right]\right)-\frac{E\left[\gamma_{e}^{2}\right]-E\left[\gamma_{e}\right]^{2}}{2\left(1+E\left[\gamma_{e}\right]\right)^{2}}\right]
$$

By substituting (53) in to (55) for $h=1, h=2$ we can obtain approximated closed form expression for ergodic capacity.

\section{High SNR analysis: outage probability}

In this section, we analyze the fixed gain relay system in high SNR. Let $C=D \rho_{1}, \rho_{2}=\mu \rho_{1}$, and $z=\Lambda / \rho_{1}$ then we can rewrite (44) as,

$$
\begin{aligned}
& F_{\gamma_{e}}(z)=1-2 \sum_{w_{o}=1}^{L}(-1)^{w_{o}+1}\left(\begin{array}{c}
L \\
w_{o}
\end{array}\right) \sum_{w_{1}=0}^{w_{o}} \sum_{w_{2}=0}^{w_{1}} \ldots \sum_{w_{n_{s}-1}=0}^{w_{n_{s}-2}} \\
& \times \prod_{v=1, w_{n_{s}}=0}^{n_{s}}\left[\frac{\phi_{v}^{n_{s}-1}}{\prod_{k=1, k \neq v}^{n_{s}}\left(\phi_{v}-\phi_{k}\right)}\right]^{w_{v-1}-w_{v}} \\
& \times\left(\begin{array}{c}
w_{v-1} \\
w_{v}
\end{array}\right) \sum_{u=1}^{n_{d}} \frac{\sigma_{u}^{n_{d}-1}}{\prod_{k=1, k \neq i}^{n_{d}}\left(\sigma_{u}-\sigma_{k}\right)} \\
& \times \sqrt{\sum_{i=1, w_{n_{s}=0}}^{n_{s}} \frac{\left(w_{i-1}-w_{i}\right)}{\phi_{i} \sigma_{u}}} \sqrt{\frac{D z}{\mu}} \\
& \times \exp \left(-\sum_{i=1, w_{n_{s}=0}}^{n_{s}} \frac{\left(w_{i-1}-w_{i}\right) z}{\phi_{i}}\right) \\
& \times K_{1}\left(2 \sqrt{\sum_{i=1, w_{n_{s}}=0}^{n_{s}} \frac{\left(w_{i-1}-w_{i}\right) D z}{\mu \phi_{i} \sigma_{u}}}\right)
\end{aligned}
$$

then (56) can be rewritten as,

$$
F_{\gamma_{e}}(z)=1-\Psi \sqrt{\frac{D z}{\mu}} \exp \left(-\beta_{5} z\right) K_{1}\left(2 \sqrt{\frac{\alpha_{5} D z}{\mu}}\right)
$$

where

$$
\begin{gathered}
\Psi=2 \sum_{w_{o}=1}^{L}(-1)^{w_{o}+1}\left(\begin{array}{c}
L \\
w_{o}
\end{array}\right) \sum_{w_{1}=0}^{w_{o}} \sum_{w_{2}=0}^{w_{1}} \cdots \sum_{w_{n_{s}-1}=0}^{w_{n_{s}-2}} \prod_{v=1, w_{n_{s}}=0}^{n_{s}} \\
\times\left[\frac{\phi_{v}^{n_{s}-1}}{\prod_{k=1, k \neq v}^{n_{s}}\left(\phi_{v}-\phi_{k}\right)}\right]^{w_{v-1}-w_{v}}\left(\begin{array}{c}
w_{v-1} \\
w_{v}
\end{array}\right) \\
\times \sum_{u=1}^{n_{d}} \frac{\sigma_{u}^{n_{d}-1}}{\prod_{k=1, k \neq i}^{n_{d}}\left(\sigma_{u}-\sigma_{k}\right)} \sqrt{\sum_{i=1, w_{n_{s}}=0}^{n_{s}} \frac{\left(w_{i-1}-w_{i}\right)}{\phi_{i} \sigma_{u}}} \\
\sum_{i=1, w_{n_{s}=0}} \frac{\left(w_{i-1}-w_{i}\right)}{\phi_{i}}
\end{gathered}
$$

and

$$
\alpha_{5}=\sum_{i=1, w_{n_{s}=0}}^{n_{s}} \frac{\left(w_{i-1}-w_{i}\right)}{\phi_{i} \sigma_{u}} .
$$

Now, we expand the exponential function using Maclaurin series and Bessel function using ([27], Equation 8.446) in (57) to obtain, 


$$
\begin{aligned}
F_{\gamma_{e}}(z)= & 1-\Psi \sqrt{\frac{D z}{\mu}} \sum_{S=0}^{\infty} \frac{\left(-\beta_{5} z\right)^{S}}{S !}\left[\frac{1}{2}\left(\frac{\alpha_{5} D z}{\mu}\right)^{\frac{-1}{2}}\right. \\
& \left.+\sum_{k=0}^{\infty}\left(\frac{\alpha_{5} D z}{\mu}\right)^{\frac{1+2 k}{2}} \frac{\Upsilon_{k}}{k !(k+1) !}\right]
\end{aligned}
$$

Now it is observed that $z^{n}, n<\min \left(L n_{s}, n_{d}\right)$ sum to zero and after limiting to high order terms with some simplifications we have,

$$
\begin{aligned}
F_{\gamma_{e}^{\infty}}(z)= & \Psi\left[\frac{(-1)^{N+1} \beta_{5}^{N}}{2 \sqrt{\alpha_{5} N !}}-\sum_{k=0}^{N-1} \frac{\left(-\beta_{5}\right)^{N-k-1} \alpha_{5}^{\frac{1+2 k}{2}}}{(N-k-1) !}\right. \\
& \left.\times\left(\frac{D}{\mu}\right)^{k+1} \frac{\Upsilon_{k}}{k !(k+1) !}\right] z^{N}+o\left(z^{N+1}\right)
\end{aligned}
$$

where $N=\min \left(L n_{s}, n_{d}\right)$ and

$$
\Upsilon_{k}=\frac{1}{2}\left[\ln \left(\frac{\alpha_{5} D z}{\mu}\right)-\psi(k+1)-\psi(k+2)\right]
$$

where $\psi(\cdot)$ is Euler Psi function. It is observed from the fixed gain asymptotic outage expression that the diversity gain of the system is similar to the csi-assisted relay scheme.

\section{High SNR analysis: average SER}

We can write the asymptotic average SER as [31],

$$
P_{s e r}^{\infty}=\frac{2^{t} a \Theta \Gamma\left(t+\frac{3}{2}\right)\left(2 b \rho_{1}\right)^{-(t+1)}}{\sqrt{\pi}}+o\left(\rho_{1}^{-(t+1)}\right)
$$

where

$$
\begin{aligned}
\Theta= & \Psi\left[\frac{(-1)^{N+1} \beta_{5}^{N}}{2 \sqrt{\alpha_{5} N !}}-\sum_{k=0}^{N-1} \frac{\left(-\beta_{5}\right)^{N-k-1} \alpha_{5}^{\frac{1+2 k}{2}}}{(N-k-1) !}\right. \\
& \left.\times\left(\frac{D}{\mu}\right)^{k+1} \frac{\Upsilon_{k}}{k !(k+1) !}\right]
\end{aligned}
$$

where $\Psi$ as in (58) and $a$ and $b$ define the modulation scheme. $t=\min \left[L n_{s}, n_{d}\right]-1$ and diversity gain $G_{d}=$ $t+1$.

\section{Numerical analysis}

Here we carry out the numerical analysis and verify our results using Monte Carlo simulations. We use the exponential correlation matrix structure where $(i, j)$ th element of the matrix $\Phi_{s}$ is $\rho_{s}^{|i-j|}$ and that of the correlation matrix $\Phi_{d}$ is $\rho_{d}^{|i-j|}$. Without loss of generality we consider $\rho_{1}=$ $\rho_{2}(\mu=1)$ in all the cases shown in the figures. Fixed gain type 1 in (49) is used. Exponential correlation matrices defined above have full rank. Hence, we obtain the desired diversity.

Figure 1 shows the outage probability variation with the average SNR of the first hop. Curves are plotted for different antenna configurations and correlation parameters. It is observed from the figure that the increase of the number of antennas, improves the outage probability. High SNR curves are also plotted where we can clearly see how diversity gain is varying. One can notice that the left three curves have a diversity gain of four and the two right most curves have a diversity gain of two. It is noticed that the increase of correlation decreases the performance. The outage probability variation for fixed gain relay is depicted in Figure 2. Here we see an improvement in the performance when the number of antennas and number of relays increase. However, as in the csi-assisted case we can observe that this improvement depends on the diversity gain. It is further observed that the increase of correlation decreases the performance. Moreover, one can notice that the csi-assisted relay outperforms the fixed gain one by approximately $3 \mathrm{~dB}$. Moreover Monte Carlo simulation results exactly match with the analytical ones.

Average SER figures are depicted in Figures 3 and 4 for csi-assisted and fixed gain relay schemes, respectively. In both figures it is observed that the increase of number of antennas and the number of relays improve the average SER. Conversely, the increase of correlation parameters decreases it. Without loss of generality we have considered the BPSK, QPSK, and QAM schemes to demonstrate the average SER variation. High SNR curves are plotted and they are compatible with the exact ones in medium to high SNR and they show the diversity gain variation. Further, we can notice that the csi-assisted relay performs better than the fixed gain relay. Monte carlo simulations coincide with the analytical ones and it shows the accuracy of our results.

Figures 5 and 6 show the ergodic capacity variation for csi-assisted and fixed gain relay cases. Without loss of generality, we fixed the number of antennas to be $n_{s}=n_{d}=2$ and $\rho_{1}=\rho_{2}=2 \mathrm{~dB}$. We have plotted the ergodic capacity variation against the number of relays to demonstrate the fact that the ergodic capacity can be improved with the increase of correlation for a higher number of relays. From the figures it is noticed that the increase of correlation at the destination $\rho_{d}$, decreases the ergodic capacity , however, the increase of correlation parameter at the source increases it. The reason for this behavior can be explained as follows; the relay to destination is a point to point link, hence, the increase of correlation decreases the ergodic capacity, however, the source to relay link is a point-to-multipoint link, hence the increase of correlation parameter reduces the channel hardening effect $[26,32]$ which results in a higher capacity. Moreover, Monte 
Carlo simulations exactly coincide with the analytical ones for csi-assisted one and are closely compatible with the approximated fixed gain ergodic capacity.

\section{Conclusion}

We have investigated the performance of a partial relay selection network with the effect of antenna correlation at the source and the destination. Two relay schemes; csi-assisted and fixed gain relay schemes have been considered and exact closed form expressions for outage probability, average SER and ergodic capacity have been derived. Our results can be used to quantify the effect of antenna correlation in partial relay selection. Further, we have provided an asymptotic analysis which can be used to obtain an insight of the system performance. In addition, we have showed that for a higher number of relays, the ergodic capacity can be improved with higher correlation at the source.

\section{Appendix 1}

Let $\gamma_{i}=\rho_{1}|| \mathbf{h}_{s i} \|_{F}$ and we rewrite (1) as,

$$
\gamma_{1}=\max _{1<i<L} \gamma_{i}
$$

We find the pdf of $\gamma_{i}$ as $[25,26]$,

$$
p_{\gamma_{i}}(z)=\sum_{i=1}^{n_{s}} \frac{\phi_{i}^{n_{s}-2}}{\prod_{k=1, k \neq i}^{n_{s}}\left(\phi_{i}-\phi_{k}\right)} \frac{\exp \left(\frac{-z}{\rho_{1} \phi_{i}}\right)}{\rho_{1}}
$$

and cdf of $\gamma_{i}$ as,

$$
F_{\gamma_{i}}(z)=1-\sum_{i=1}^{n_{s}} \frac{\phi_{i}^{n_{s}-1}}{\prod_{k=1, k \neq i}^{n_{s}}\left(\phi_{i}-\phi_{k}\right)} \exp \left(\frac{-z}{\rho_{1} \phi_{i}}\right)
$$

We assume that the relays are distributed homogeneously such that they have equal average SNR, further, we assume that the $S-R_{i} \forall i$ channels are independent. Then we can derive the cdf of $\gamma_{1}$ as,

$$
\begin{aligned}
F_{\gamma_{1}}(z)= & {\left[F_{\gamma_{i}}(z)\right]^{L}=\left[1-\sum_{i=1}^{n_{s}} \frac{\phi_{i}^{n_{s}-1}}{\prod_{k=1, k \neq i}^{n_{s}}\left(\phi_{i}-\phi_{k}\right)}\right.} \\
& \left.\times \exp \left(\frac{-z}{\rho_{1} \phi_{i}}\right)\right]^{L}
\end{aligned}
$$

Using multinominal theorem and after some simplifications, we derive $F_{\gamma_{1}}(z)$ as in (8),

\section{Appendix 2}

\section{Outage probability}

Pdf of $\gamma_{2}$ can be obtained from (6) and the cdf of $\gamma_{1}$ is derived in (8). Following the same procedure as mentioned in ([4], Appendix A), $F_{\gamma_{e}}(\Lambda)$ can be expressed as,

$$
F_{\gamma_{e}}(\Lambda)=1-\int_{0}^{\infty} \tilde{F}_{\gamma_{1}}\left(\Lambda+\frac{\Lambda(\Lambda+1)}{z}\right) p_{\gamma_{2}}(z+\Lambda) d z
$$

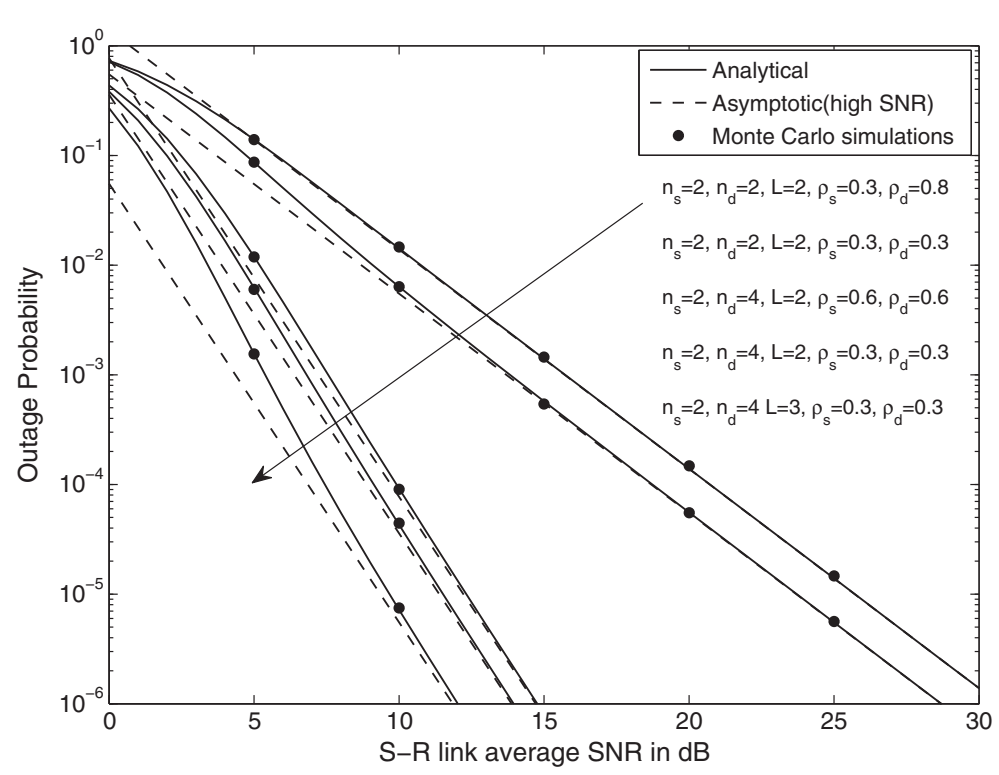

Figure 1 Csi-assisted outage probability. 


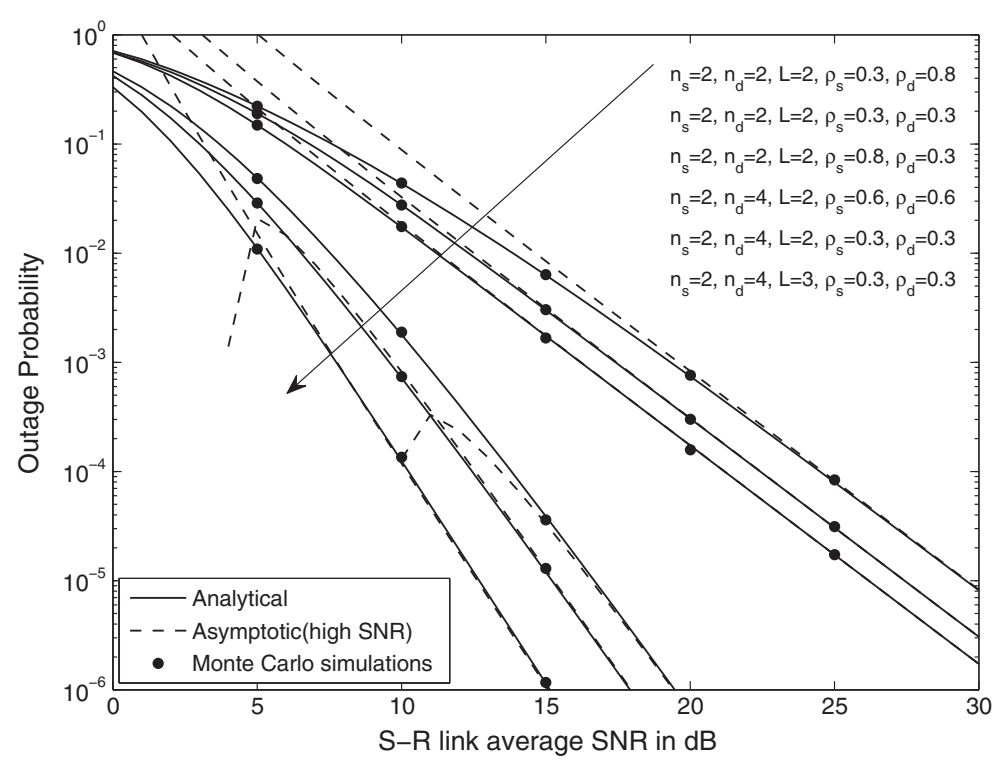

Figure 2 Fixed Gain outage probability.

where $\tilde{F}_{\gamma_{1}}(z)=1-F_{\gamma_{1}}(z)$. By substituting (8) and (6) to $(70)$ and by mathematical simplifications we obtain the $F_{\gamma_{e}}(\Lambda)$ as,

$$
\begin{aligned}
F_{\gamma_{\mathrm{e}}}(\Lambda)= & 1-\sum_{w_{0}=1}^{L}(-1)^{w_{0}+1}\left(\begin{array}{c}
L \\
w_{0}
\end{array}\right) \sum_{w_{1}=0}^{w_{0}} \sum_{w_{2}=0}^{w_{1}} \ldots \sum_{w_{n_{s}-1}=0}^{w_{n_{s}-2}} \\
& \times \prod_{i=1, w_{n_{\mathrm{s}}}=0}^{n_{s}}\left[\frac{\phi_{i}^{n_{s}-1}}{\prod_{k=1, k \neq i}^{n_{s}}\left(\phi_{i}-\phi_{k}\right)}\right]^{w_{i-1}-w_{i}}\left(\begin{array}{c}
w_{i-1} \\
w_{i}
\end{array}\right)
\end{aligned}
$$

$$
\begin{aligned}
& \times \sum_{u=1}^{n_{d}} \frac{\sigma_{u}^{n_{d}-1} e^{-\frac{\Lambda}{\rho_{2} \sigma_{u}}-\sum_{i=1, w_{n_{s}}=0}^{n_{S}} \frac{\left(w_{i-1}-w_{i}\right) \Lambda}{\rho_{1} \phi_{i}}}}{\prod_{k=1, k \neq u}^{n_{d}}\left(\sigma_{u}-\sigma_{k}\right) \rho_{2} \sigma_{u}} \\
& \times \int_{0}^{\infty} e^{-\frac{z}{\rho_{2} \sigma_{u}}-\sum_{i=1, w_{n_{s}}=0}^{n_{S}} \frac{\left(w_{i-1}-w_{i}\right) \Lambda(\Lambda+c)}{z \rho_{1} \phi_{i}}} d z
\end{aligned}
$$

Performing the integration with the help of ([27], 3.471.9), we can obtain the closed form solution as in (12).

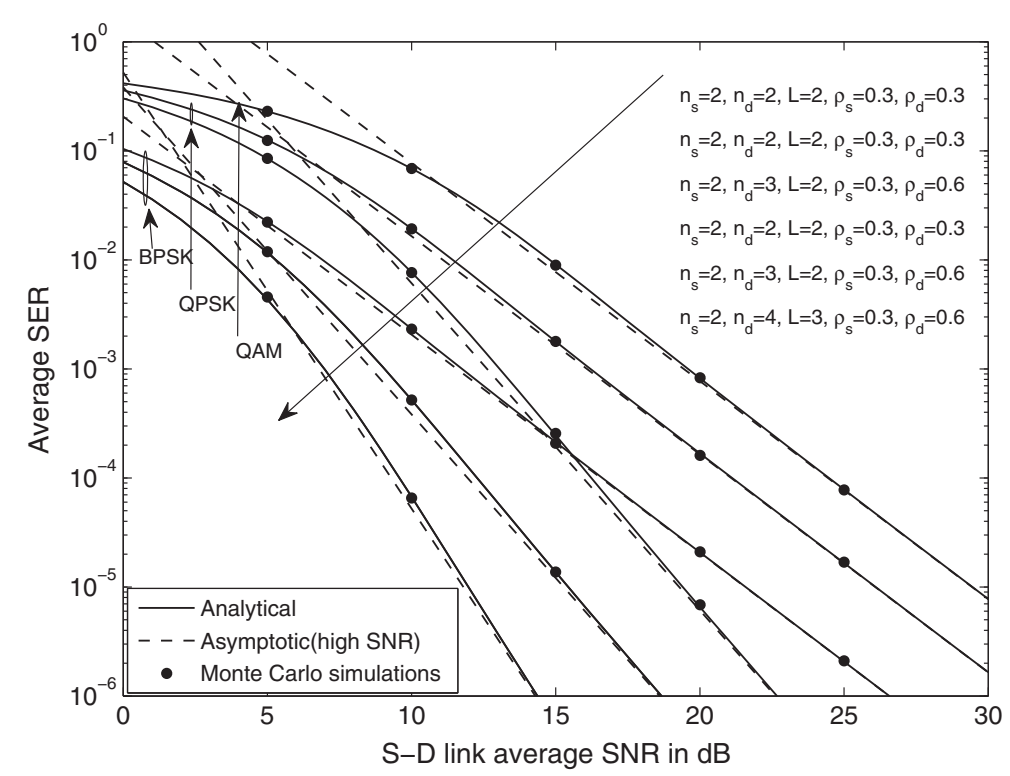

Figure 3 Symbol error rate, Csi-assisted systems. 


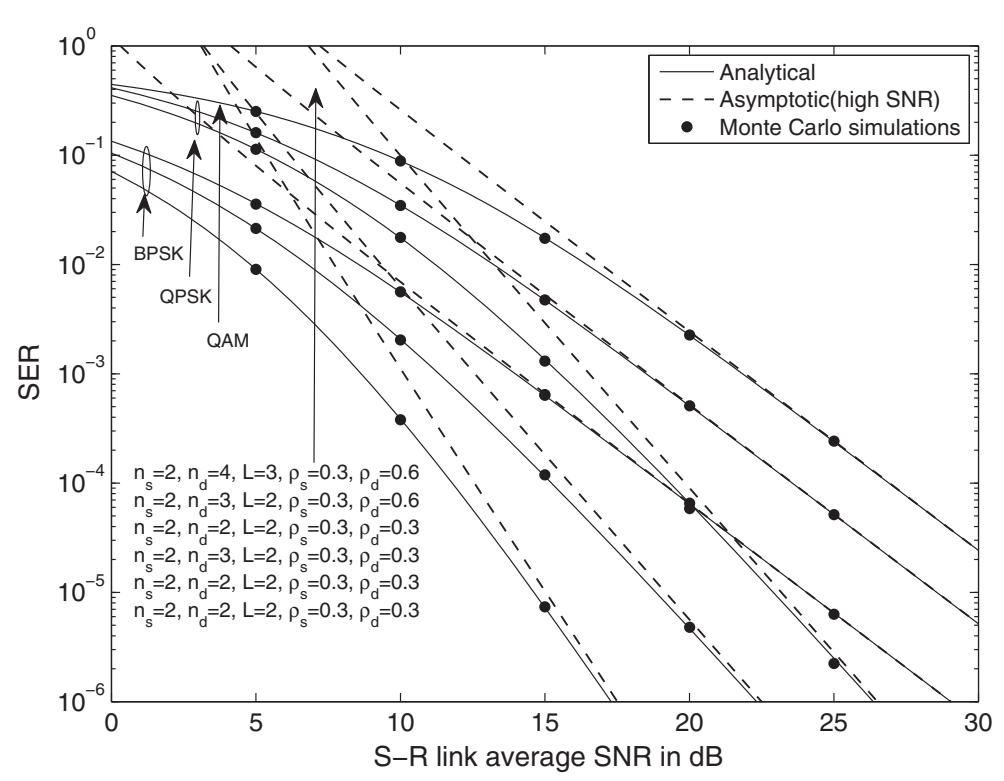

Figure 4 Symbol error rate, fixed gain systems.

\section{Ergodic capacity}

We can rewrite (20) as,

$$
C_{\mathrm{erg}}=\frac{R}{\ln 2} E\left[\ln \left(1+\frac{\gamma_{1} \gamma_{2}}{\gamma_{1}+\gamma_{2}+1}\right)\right]
$$

After some mathematical manipulations [33],

$$
C_{\mathrm{erg}}=\frac{R}{\ln 2} E\left[\ln \left(\frac{\left(1+\gamma_{1}\right)\left(1+\gamma_{2}\right)}{1+\gamma_{1}+\gamma_{2}}\right)\right]
$$

Now, we can rewrite (73)

$$
C_{\text {erg }}=\frac{R}{\ln 2}\left[E\left[\ln \left(1+\gamma_{1}\right)\right]+E\left[\ln \left(1+\gamma_{2}\right)\right]-E\left[\ln \left(1+\gamma_{3}\right)\right]\right]
$$

where $\gamma_{3}=\gamma_{1}+\gamma_{2}$. Now $\chi_{2}=E\left[\ln \left(1+\gamma_{2}\right)\right]$ can be derived using pdf of $\gamma_{2}$ as,

$$
\chi_{2}=\int_{0}^{\infty}(1+z) \sum_{u=1}^{n_{d}} \frac{\sigma_{u}^{n_{d}-2}}{\prod_{k=1, k \neq u}^{n_{d}}\left(\sigma_{u}-\sigma_{k}\right)} \frac{e^{\frac{-z}{\rho_{2} \sigma_{u}}}}{\rho_{2}} d z
$$

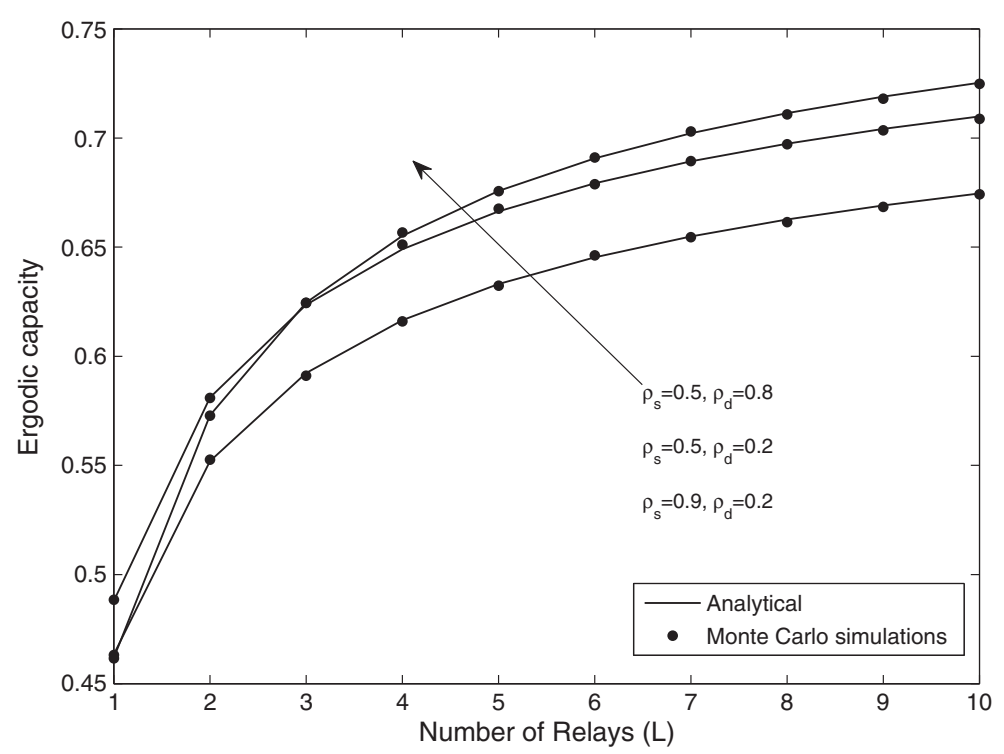

Figure 5 Ergodic capacity, csi-assisted systems. 


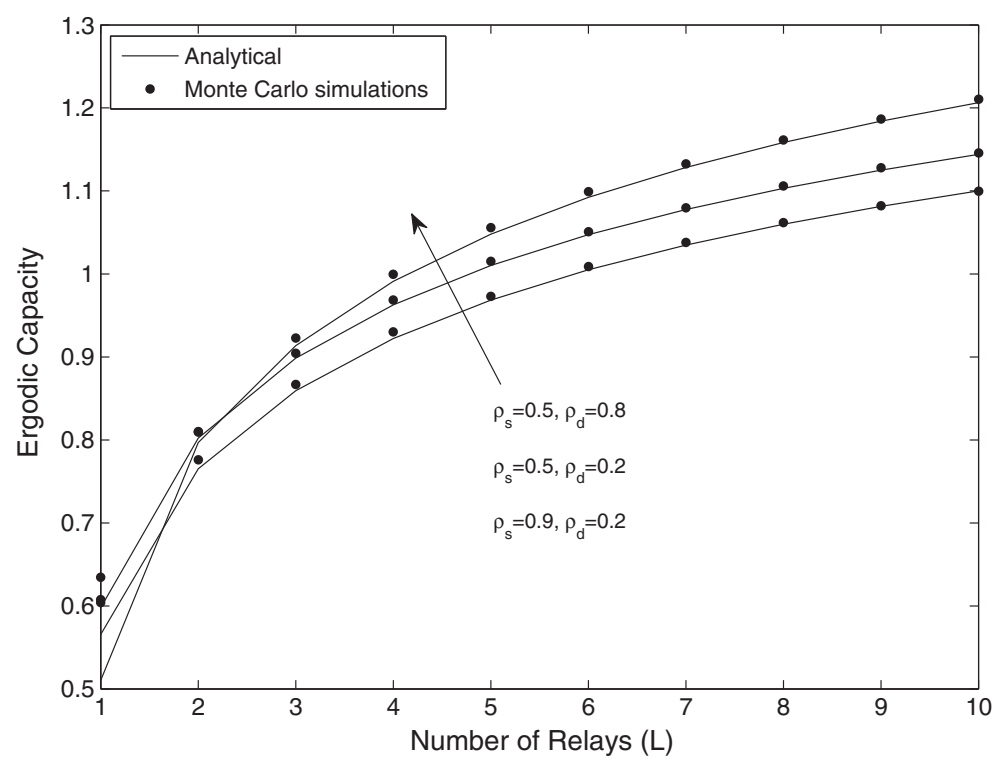

Figure 6 Ergodic capacity, fixed gain systems.

Performing the integration with the help of ([27], Equation 4.337.5) we obtain the closed form expression for $\chi_{2}$ as in (23). Similarly, we can derive $\chi_{1}=E\left[\ln \left(1+\gamma_{1}\right)\right]$ as in (22). Performing convolution operation we obtain the pdf of $\gamma_{3}=\gamma_{1}+\gamma_{2}$ as,

$$
p_{\gamma_{3}}(z)=\int_{0}^{z} p_{\gamma_{2}}(t) p_{\gamma_{1}}(z-t) d t
$$

We carry out the integration to obtain $p_{\gamma_{3}}(z)$ as,

$$
p_{\gamma_{3}}(z)=\left\{\begin{array}{l}
\delta_{1}(z), \frac{1}{\rho_{2} \sigma_{u}} \neq \frac{\beta}{\rho_{1}} \\
\delta_{2}(z), \frac{1}{\rho_{2} \sigma_{u}}=\frac{\beta}{\rho_{1}}
\end{array}\right.
$$

where

$$
\begin{aligned}
\delta_{1}(z)= & \sum_{u=1}^{n_{d}} \frac{\sigma_{u}^{n_{d}-1}}{\prod_{k=1, k \neq u}^{n_{d}}\left(\sigma_{u}-\sigma_{k}\right)} \sum_{w_{0}=1}^{L}(-1)^{w_{0}+1}\left(\begin{array}{c}
L \\
w_{0}
\end{array}\right) \\
& \times \sum_{w_{1}=0}^{w_{0}} \sum_{w_{2}=0}^{w_{1}} \ldots \sum_{w_{n_{s}-1}=0}^{w_{n_{s}-2}} \prod_{i=1, w_{n_{s}}=0}^{n_{s}} \\
& \times\left[\frac{\phi_{i}^{n_{s}-1}}{\prod_{k=1, k \neq i}^{n_{s}}\left(\phi_{i}-\phi_{k}\right)}\right]^{w_{i-1}-w_{i}}\left(\begin{array}{c}
w_{i-1} \\
w_{i}
\end{array}\right) \\
& \times\left(\frac{1}{\rho_{2} \sigma_{u}}-\frac{\beta}{\rho_{1}}\right)^{-1} \frac{\beta}{\rho_{2} \sigma_{u} \rho_{1}}\left(e^{-\frac{z \beta}{\rho_{1}}}-e^{-\frac{z}{\rho_{2} \sigma_{u}}}\right)
\end{aligned}
$$

and

$$
\begin{aligned}
\delta_{2}(z)= & \sum_{u=1}^{n_{d}} \frac{\sigma_{u}^{n_{d}-1}}{\prod_{k=1, k \neq u}^{n_{d}}\left(\sigma_{u}-\sigma_{k}\right)} \sum_{w_{0}=1}^{L}(-1)^{w_{0}+1}\left(\begin{array}{c}
L \\
w_{0}
\end{array}\right) \\
& \times \sum_{w_{1}=0}^{w_{0}} \sum_{w_{2}=0}^{w_{1}} \ldots \sum_{w_{n_{s}-1}=0}^{w_{n_{s}-2}}
\end{aligned}
$$

$$
\begin{aligned}
& \times \prod_{i=1, w_{n_{s}}=0}^{n_{s}}\left[\frac{\phi_{i}^{n_{s}-1}}{\prod_{k=1, k \neq i}^{n_{s}}\left(\phi_{i}-\phi_{k}\right)}\right]^{w_{i-1}-w_{i}} \\
& \times\left(\begin{array}{c}
w_{i-1} \\
w_{i}
\end{array}\right) \frac{\beta}{\rho_{2} \sigma_{u} \rho_{1}} z e^{-\frac{z}{\rho_{2} \sigma_{u}}}
\end{aligned}
$$

Now by using the same procedure as in the derivation of $\chi_{2}$, we can obtain the closed form expression for $\chi_{3}=$ $E\left[\ln \left(1+\gamma_{3}\right)\right]$. We use $\chi_{1}, \chi_{2}$, and $\chi_{3}$ to get the closed form expression for the ergodic capacity as in (21).

\section{Appendix 3}

\section{Outage probability}

Then following the same procedure as mentioned in ([23], Equation 5) CDF of $\gamma_{e}$ can be calculated as,

$$
F_{\gamma_{e}}(\Lambda)=\int_{0}^{\infty} P\left(\gamma_{1}<\Lambda+\frac{C \Lambda}{z} \mid \gamma_{2}\right) p_{\gamma_{2}}(z) d z
$$

Substituting (6) and (8) into (80) and after some algebraic manipulations, 


$$
\begin{aligned}
F_{\gamma_{\mathrm{e}}}(\Lambda)= & 1-2 \sum_{w_{o}=1}^{L}(-1)^{w_{o}+1}\left(\begin{array}{c}
L \\
w_{o}
\end{array}\right) \sum_{w_{1}=0}^{w_{o}} \sum_{w_{2}=0}^{w_{1}} \ldots \sum_{w_{n_{s}-1}=0}^{w_{n_{s}-2}} \\
& \times \prod_{v=1, w_{n_{s}}=0}^{n_{s}}\left[\frac{\phi_{v}^{n_{s}-1}}{\prod_{k=1, k \neq v}^{n_{s}}\left(\phi_{v}-\phi_{k}\right)}\right]^{w_{v-1}-w_{v}} \\
& \times\left(\begin{array}{c}
w_{v-1} w_{v} \\
w_{v}
\end{array} \sum_{u=1}^{n_{d}} \frac{\sigma_{u}^{n_{D}-1}}{\prod_{k=1, k \neq i}^{n_{d}}\left(\sigma_{u}-\sigma_{k}\right) \rho_{2} \sigma_{u}}\right. \\
& \times \exp \left(-\sum_{i=1, x_{n_{s}=0}}^{n_{s}} \frac{\left(w_{i-1}-w_{i}\right) \Lambda}{\rho_{1} \phi_{i}}\right) \\
& \times \int_{0}^{\infty} \exp \left(\frac{-z}{\rho_{2} \sigma_{u}}-\sum_{i=1, w_{n_{s}=0}}^{n_{s}} \frac{\left(w_{i-1}-w_{i}\right)}{z \rho_{1} \phi_{i}} \Lambda C\right) d z
\end{aligned}
$$

Using ([27], Equation 3.471.9) the closed form for outage probability can be expressed as in (44).

\section{Competing interests}

The authors declare that they have no competing interests.

\section{Acknowledgements}

This research was supported by the Finnish Funding Agency for Technology and Innovation (Tekes), Renesas Mobile, Nokia Siemens Networks, Elektrobit.

Received: 13 April 2012 Accepted: 12 July 2012

Published: 16 August 2012

\section{References}

1. JN Laneman, DNC Tse, GW Wornell (eds), Cooperative diversity in wireless networks: Efficient protocols and outage behavior. IEEE Trans. Inf. Theory. 50, 3062-3080 (2004)

2. MO Hasna, M Alouini (eds), End-to-end performance of transmission systems with relays over Rayleigh-fading channels. IEEE Trans. Wirel. Commun. 2, 1126-1131 (2003)

3. MO Hasna, M Alouini (eds), A performance study of dual-hop transmissions with fixed gain relays. IEEE Trans. Wirel. Commun. 3, 1963-1968 (2004)

4. D Senarathne, C Tellambura (eds), Unified Exact Performance Analysis of Two-Hop Amplify-and-Forward Relaying in Nakagami Fading. IEEE Trans. Veh. Technol. 59, 1529-1534 (2010)

5. RHY Louie, Y Li, B Vucetic (eds), Perform. Anal. Beamforming in Two Hop Amplify and Forward Relay Networks (IEEE ICC, Beijing, China, 2008)

6. DB da Costa, S Aïssa (eds), Beamforming in Dual-Hop Fixed Gain Relaying Syst (IEEE, Dresden, Germany, 2009)

7. RHY Louie, Y Li, HA Suraweera, B Vucetic (eds), Performance analysis of beamforming in two hop amplify and forward relay networks with antenna correlation. IEEE Trans. Wirel. Commun. 8, 3131-3142 (2009)

8. DB da Costa, S Aïssa (eds), Cooperative dual-hop relaying systems with beamforming over Nakagami-m fading channels. IEEE Trans. Wirel. Commun. 8, 3950-3954 (2009)

9. NS Ferdinand, N Rajatheva (eds), Unified performance analysis of two-hop amplify-and-forward relay systems with antenna correlation. IEEE Trans. Wirel. Commun. 10, 3002-3011 (2011)

10. G Amarasuriya, C Tellambura, M Ardakani (eds), Impact of antenna correlation on a new dual-hop MIMO AF relaying model, vol. 2010 (Article ID 956721, 2010), pp. 1-14

11. TQ Duong, GC Alexandropoulos, TA Tsiftsis, HJ Zepernick (eds), Orthogonal space-time block codes with CSI-assisted amplify-and-forward relaying in correlated Nakagami- $m$ fading channels. IEEE Trans. Veh. Technol. 60, 882-889 (2011)

12. HA Suraweera, HK Garg, A Nallanathan (eds), Beamforming in Dual-Hop Fixed Gain Relay Systems with Antenna Correlation (IEEE ICC, Cape Town, South Africa, 2010)

13. A Bletsas, H Shin, MZ Win (eds), Cooperative communications with outage-optimal opportunistic relaying. IEEE Trans. Wirel. Commun. 6, 3450-3460 (2007)

14. B Barua, $\mathrm{H} \mathrm{Ngo}, \mathrm{H}$ Shin (eds), On the SEP of cooperative diversity with opportunistic relaying. IEEE Commun. Lett. 12, 727-729 (2008)

15. I Krikidis, J Thompson, S McLaughlin, N Goertz (eds), Amplify-and-forward with partial relay selection. IEEE Commun. Lett. 12, 235-237 (2008)

16. DB da Costa, S Aïssa (eds), End-to-end performance of dual-hop semi-blind relaying systems with partial relay selection. IEEE Trans. Wirel. Commun. 8, 4306-4315 (2009)

17. DB da Costa, S Aïssa (eds), Performance analysis of relay selection techniques with clustered fixed-gain relays. IEEE Signal Process. Lett 17, 201-204 (2010)

18. DB da Costa, S Aïssa (eds), Capacity analysis of cooperative systems with relay selection in Nakagami-m Fading. IEEE Commun. Lett. 13, 637-639 (2009)

19. DB da Costa, S Aïssa (eds), Amplify-and-forward relaying in channel-noise-assisted cooperative networks with relay selection. IEEE Commun. Lett. 14, 608-610 (2010)

20. M Torabi, D Haccoun (eds), Capacity analysis of opportunistic relaying in cooperative systems with outdated channel information. IEEE Commun. Lett. 14, 1137-1139 (2010)

21. HA Suraweera, M Soysa, C Tellambura, HK Garg (eds), Performance analysis of partial relay selection with feedback delay. IEEE Signal Process. Lett. 17, 531-534 (2010)

22. JL Vicario, A Bel, JA Lopez-Salcedo, G Seco (eds), Opportunistic relay selection with outdated CSI: outage probability and diversity analysis. IEEE Trans. Wirel. Commun. 8, 2872-2876 (2009)

23. DS Michalopoulos, HA Suraweera, GK Karagiannidis, R Schober (eds), Amplify-and-Forward Relay Sel. with Outdated Channel State Inf (IEEE Globecom, Miami, Florida, 2010)

24. TKY Lo (ed.), Maximum ratio transmission. IEEE Trans. Commun. 47, 1456-1461 (1999)

25. L Musavian, M Dohler, MR Nakhai, AH Aghvami (eds), Closed-form capacity expressions of orthogonalized correlated MIMO channels. IEEE Commun. Lett. 8, 365-367 (2004)

26. NS Ferdinand, N Rajatheva, M Latva-aho (eds), Effect of Antenna Correlation on the Performance of MIMO Multi-User Dual Hop Relay Network (IEEE Globecom, Houston, Texas, 2011)

27. IS Gradshteyn, IM Ryzhik, Table of Integrals, Ser. Prod (Elsevier, New York, 2007)

28. MK Simon, MS Alouini, Digital Commun. over Fading Channels: A Unified Approach to Perform. Anal (John Wiley and Sons, New York, 2010)

29. J Proakis, Digital Commun (McGraw Hill, New York, 2001)

30. Z Fang, L Li, Z Wang (eds), Asymptotic performance analysis of multihop relayed transmissions over Nakagami-m fading channels. IEICE Trans. Commun. E91-B, 4081-4084 (2008)

31. Z Wang, GB Giannakis (eds), A simple and general parameterization quantifying performance in fading channels. IEEE Trans. Commun. 51, 1389-1398 (2003)

32. D Park, SY Park (eds), Performance analysis of multi-user diversity under transmit antenna correlation. IEEE Trans. Commun. 56, 666-674 (2008)

33. L Fan, X Lei, W Li (eds), Exact closed-form expression for ergodic capacity of amplify-and-forward relaying in channel-noise-assisted cooperative networks with relay selection. IEEE Commun. Lett. 15, 332-333 (2011)

doi:10.1186/1687-1499-2012-261

Cite this article as: Ferdinand et al:: Impact of antenna correlation on the performance of partial relay selection. EURASIP Journal on Wireless Communications and Networking 2012 2012:261. 